\title{
The Brain in Its Body: Motor Control and Sensing in a Biomechanical Context
}

\author{
Hillel J. Chiel, ${ }^{1,2,3}$ Lena H. Ting, ${ }^{4}$ Örjan Ekeberg, ${ }^{5}$ and Mitra J. Z. Hartmann ${ }^{6,7}$ \\ Departments of ${ }^{1}$ Biology, ${ }^{2}$ Neurosciences, and ${ }^{3}$ Biomedical Engineering, Case Western Reserve University, Cleveland, Ohio 44106-7080, ${ }^{4}$ Department of \\ Biomedical Engineering, Emory University and Georgia Institute of Technology, Atlanta, Georgia 30332-0535, ${ }^{5}$ Department of Computational Biology, \\ School of Computer Science and Communication, Royal Institute of Technology S-10044 Stockholm, Sweden, and Departments of ${ }^{6}$ Biomedical and \\ ${ }^{7}$ Mechanical Engineering, Northwestern University, Evanston, Illinois 60208
}

\begin{abstract}
Although it is widely recognized that adaptive behavior emerges from the ongoing interactions among the nervous system, the body, and the environment, it has only become possible in recent years to experimentally study and to simulate these interacting systems. We briefly review work on molluscan feeding, maintenance of postural control in cats and humans, simulations of locomotion in lamprey, insect, cat and salamander, and active vibrissal sensing in rats to illustrate the insights that can be derived from studies of neural control and sensing within a biomechanical context. These studies illustrate that control may be shared between the nervous system and the periphery, that neural activity organizes degrees of freedom into biomechanically meaningful subsets, that mechanics alone may play crucial roles in enforcing gait patterns, and that mechanics of sensors is crucial for their function.
\end{abstract}

\section{Introduction}

In his classic studies of human locomotion, the great Russian physiologist Nikolai Bernstein noted that neural control using purely feedforward commands would not be sufficient for animals to deal with changing contingencies in the environment because of the unpredictable nature of environmental loads. In addition, the large number of degrees of freedom about the joints, the complex kinematic chains due to the structure of the organism, and inertial forces all complicated the ability of the nervous system to generate appropriate commands. At the same time, by visualizing actual movements during locomotion, he noted regular, highly coordinated biodynamic waves, i.e., characteristic groupings of kinematic variables. These characteristic groupings provided an accurate characterization of walking across many individuals, and could also clarify the development of locomotion from childhood to adulthood, as well as the decay of locomotion with age. The implication of these results was that the nervous system grouped together degrees of freedom. Moreover, locomotion was not purely due to neural activity, but the biomechanics of the body and interactions with the environment (Bernstein, 1967).

How can the nervous system group together degrees of freedom? How can the nervous system generate robust behavior in the face of continuously changing environmental conditions? How can behavior flexibly change in response to appropriate sensory input? As an animal moves through its environment,

Received July 10, 2009; revised Aug. 4, 2009; accepted Aug. 17, 2009.

This work was supported by National Institutes of Health Grants NS047073 (H.J.C.) and HD-046922 and NS053822 (L.H.T.), the Seventh European Framework Program, project LAMPETRA (ICT-2007.8.3-FET) (0.E.), and National Science Foundation Grants IIS-0613568, IOS-0818414, and IOS-0846088 (M.J.Z.H.). We thank an anonymous reviewer for helpful comments on an earlier draft of this manuscript.

Correspondence should be addressed to Dr. Hillel J. Chiel, Department of Biology, Case Western Reserve University, 2080 Adelbert Road, Cleveland, OH 44106-7080. E-mail: hj@@case.edu.

D01:10.1523/JNEUROSCI.3338-09.2009

Copyright $\odot 2009$ Society for Neuroscience $\quad$ 0270-6474/09/2912807-08\$15.00/0 how do the dynamics of its periphery affect the process of sensing? These questions continue to engage researchers interested in motor and sensory systems. An implication of Bernstein's observations was that adaptive behavior could best be understood by looking at the interaction of the nervous system, the biomechanics of the body, and the complex dynamics of the environment (Chiel and Beer, 1997).

Advances in the ability to measure, simulate, and control biomechanics and neural control have made it feasible to use this broader framework for the analysis of motor and sensory systems. To illustrate the power of these new approaches, we review recent work that uses new computational, theoretical, and experimental tools to understand the interaction of biomechanics and neural control in feeding behavior, postural control, locomotion and sensing.

\section{Manipulating neuromechanical equilibrium points for multifunctionality}

Multifunctionality is a ubiquitous property of biological systems. We can use our legs to walk forwards and backwards, to run, or to dance. Our tongues participate in feeding, respiration, and talking. We can use our hands to throw a ball, type, or for sign language. In all of these behaviors, we flexibly deploy the multiple degrees of freedom of our body to generate qualitatively different behaviors using the same peripheral structures. What are the biomechanical and neural mechanisms of multifunctionality?

One way to address this question is to find a multifunctional model system in which the nervous system, the biomechanics, and the interactions with the environment are all tractable to experimental analysis. These requirements have served as a rationale for studies of multifunctionality in the marine mollusk Aplysia californica, which uses its feeding apparatus for biting, swallowing, rejection, grazing and egg laying (Kupfermann, 1970, 1974; Kupfermann and Carew, 1974; Elliott and Susswein, 2002). 
Feeding responses in Aplysia use the same musculature and neural networks for qualitatively different functions. Biting is an attempt to grasp food. Swallowing occurs as an animal ingests food, and rejection occurs as animals encounter material that is not edible (Kupfermann, 1974). Rejection movements may also serve to reposition edible food so that animals may more effectively ingest it (Katzoff et al., 2006). In all three behaviors, the grasper must be pushed toward the jaw (protracted), and then pushed back toward the esophagus (retracted), and it must open or close at the appropriate time.

During ingestive behaviors, the radula protracts open, closes near the peak of protraction, and then retracts closed. In contrast, during rejection, the grasper closes on inedible material as it protracts, pushing the material out, and then retracts open. In general, bites may be distinguished from swallows by their different functional requirements: to grasp effectively, the protraction phase of biting is strong; if food has not been grasped, the retraction phase of biting is weaker and rapidly succeeded by another bite. In contrast, during swallowing, protraction is weak, so as not to push food out of the buccal cavity. The retraction phase of swallowing is much stronger, since this is the power stroke of the behavior that pulls food into the buccal cavity (Neustadter et al., 2002a, 2007; Novakovic et al., 2006).

What are the key control variables underlying the multifunctional feeding behavior in Aplysia? To answer this question, several concepts need to be defined.

If a system reaches a state such that, as long as it remains in that state, it will not change over time, it is at an equilibrium point (Strogatz, 1994). In a mechanical system, if forces balance one another, the state of the system is a mechanical equilibrium point. Motor control theorists have proposed that the nervous system uses mechanical equilibrium points as the basis for the control of movements: a neural command establishes a specific force/length relationship for a muscle, leading the muscle to assume a particular mechanical equilibrium position in the presence of an external load; in general, neural activation of multiple muscles will lead to specific configurations that constitute mechanical equilibrium positions if the neural input is "frozen" at its current value (Latash, 2008). Changes in neural activation or changes in external load may alter the mechanical equilibrium point and determine the trajectory of movements (Bizzi et al., 1992; Feldman et al., 1998; Latash, 2008). This hypothesis has been controversial and has been subjected to a variety of empirical tests (Feldman and Latash, 2005), and alternatives such as stochastic optimal feedback control have been proposed and tested (Nagengast et al., 2009).

It may be useful to distinguish a neuromechanical equilibrium point from a mechanical equilibrium point. A neuromechanical equilibrium point occurs when mechanical forces balance and neural output does not change. Clearly, if a system reached such a state, it would remain there, which would not be of much use behaviorally. However, if slow processes in the nervous system destabilized the neuromechanical equilibrium point, or if there were some perturbations (e.g., noise or sensory inputs to the nervous system, small mechanical perturbations to the periphery) that would lead the system state away from the equilibrium point, then behavior could continue. Identifying neuromechanical equilibrium points could organize the layout of a system's behavioral trajectories, even if the system never actually reached these states.

Several lines of evidence suggest that neuromechanical equilibrium points are key control variables for feeding in Aplysia. First, the feeding apparatus is low in mass and moves slowly, so that forces due to velocity and position, rather than inertial forces, dominate the response of system, allowing it to reach the mechanical equilibria defined by neural activation (Sutton et al., 2004). Second, the system moves toward or away from neuromechanical equilibrium points that are defined by long lasting (i.e., essentially unchanging) patterns of neural activation. By using magnetic resonance imaging of intact, behaving animals, we characterized the neuromechanical equilibrium points kinematically during feeding behaviors, i.e., the muscular configurations toward which the system moved near the end of the long-lasting patterns of neural activation (Fig. 1) (Neustadter et al., 2002a,b). 


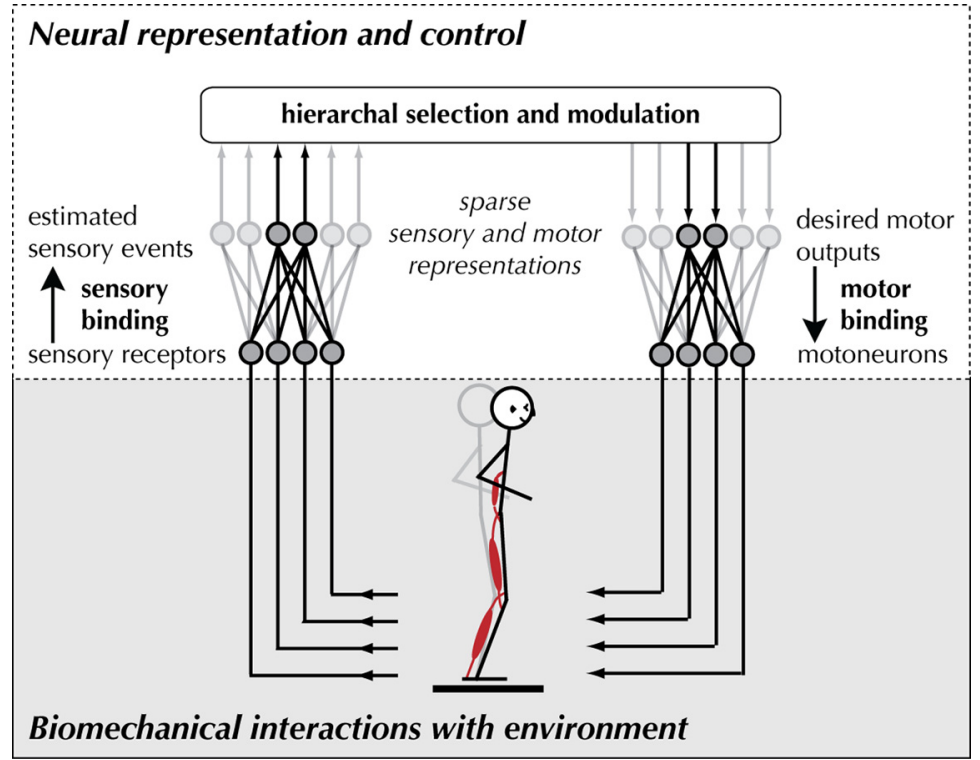

Figure 2. Neuromechanical interactions underlying neural control of movement. For any desired motor output, multiple muscles across the body need to be coordinated. Muscle synergies may help to solve this motor binding problem by providing a library of available motor reconfiguration patterns that may be selected based on the particular movement context. Like musical chords, each muscle can be activated by different muscle synergies, and there are more possible combinations for reconfiguration than muscles. Principles of sparse coding similarly predict that combinations of sensory inputs are decoded by selecting a small number of neurons from among many possible (Olshausen and Field, 2004). Current research has focused only on the lowest level of muscle synergy organization for movement, but similar neural representations and constraints on movement may also exist in the hierarchal neural control mechanisms regulating selection and temporal modulation of muscle synergies.

Third, stimulating individual motor neurons, or the entire motor pool for key muscles induces the isolated feeding apparatus to move to these different positions, at which the system remains as long as the neural activation pattern remains unchanged (Morton and Chiel, 1993b; Ye et al., 2006a,b). Fourth, kinetic models of the feeding apparatus based on experimental measurements of the biomechanics of the muscles and in vivo kinematics move to the same positions in response to prolonged neural inputs (Sutton et al., 2004; Novakovic et al., 2006). Furthermore, it appears likely that the nervous system manipulates these neuromechanical equilibrium points as it generates behavior. First, we and others have identified key interneurons in the feeding system whose primary function is to activate or inhibit motor neurons during the different phases of feeding for long periods of time, sufficient to allow the feeding apparatus to move toward or away from specific neuromechanical equilibrium points (Jing and Weiss, 2001, 2002; Ye et al., 2006b). Second, in isolated ganglia, in which sensory feeding is absent, the duration and intensity of each pattern of neural activity is prolonged (Morton and Chiel, 1993a,b). In the context of the body, proprioceptive or external sensory feedback would trigger transitions from one neuromechanical equilibrium point to the next. In the isolated ganglion, intrinsic or slow synaptic interactions allow the transitions to occur, but much more slowly, suggesting that a major function of the pattern generator is to create the long-lasting neural patterns that move the system to neuromechanical equilibrium points.

Analysis of the feeding behavior of Aplysia demonstrates that neuromechanical equilibrium points are generated by shifting coalitions of muscles (Ye et al., 2006b). For example, the protraction phase of swallowing involves the balance of forces in the I2 protractor, and the resisting forces in the hinge, interdigitating fibers that connect the grasper to the other muscles of the buccal mass. The protraction phase of biting also involves the posterior of the I3 jaw muscle, which creates a more anterior equilibrium point. In rejection, the close of the grasper (due to contraction of the I4 muscle) to push out inedible material enhances the ability of the I 2 to protract the grasper (Novakovic et al., 2006; Ye et al., 2006a; Neustadter et al., 2007).

Patterns of motor neuronal activation in vivo and in vitro not only contribute to the neuromechanical equilibrium points, but also allow animals to shift the amount of effort taken to reach them (e.g., rapidly increasing motor outflow during the retraction phase of swallowing to respond to a sudden increase in mechanical load), and enable them to flexibly shift from one sequence to another as conditions change (e.g., from biting to swallowing as food is encountered, or from swallowing to rejection in response to inedible material). Yoking together degrees of freedom by the motor programs also provides evidence for motor synergies in this system. Using a novel technique for stimulating individual identified neurons extracellularly ( $\mathrm{Lu}$ et al., 2008), it should be possible to clarify the roles of motor neurons and key interneurons in the control of neuromechanical equilibrium points in the future (Jing and Weiss, 2001, 2002). It is likely that these studies will provide insights into the control of multifunctionality in more complex animals and in humans.

\section{Neuromechanical interactions underlying muscle synergy control of posture and movement}

During standing balance control, all of the body segments are coordinated to maintain the body's center of mass (CoM) over the base of support. However, there is no one-to-one mapping between the task-level goal of controlling the CoM, and the execution-level neural commands modulating individual muscle activity and joint motions. While "postural control" and "balance control" are often used interchangeably, standing unconstrained in a gravity environment is more than simply a matter of maintaining a particular postural configuration (Nashner, 1976, 1977; Carpenter et al., 1999; Ting and Macpherson, 2004; Ting et al., 2009). When balance is perturbed, the muscles and body segments must be coordinated differently depending on the direction of the disturbance, and diverse movement patterns can be used such as swaying with feet in place, taking a step, or grabbing a handhold (Horak and Macpherson, 1996).

Because of the vast redundancy and complex biomechanical interactions in multijointed systems, the nervous system must solve a motor "binding problem" to produce consistent and predictable actions in the environment. In sensory systems, the binding problem refers to the process by which many highly variable sensory inputs are transformed into the identification of a unitary object or event in the outside world. In motor systems, a desired movement intention must be transformed into a complex spatiotemporal pattern of motor activity (Fig. 2). The activity of a single muscle can accelerate all of the joints in the body (Zajac and Gordon, 1989; Zajac, 1993), so that even simple tasks require the coordination of muscles throughout the body. Anatomically re- 
alistic biomechanical models of the cat hindlimb demonstrate that the direction of force produced at the foot by activating single leg muscle can vary by as much as $180^{\circ}$ depending on the torques produced at joints proximal and distal to that muscle (van Antwerp et al., 2007). However, the same endpoint force vector can be produced by a wide variety of muscle activation patterns (Bunderson et al., 2008). Therefore, while the biomechanical considerations for multijoint coordination present a "problem" to the nervous system, the redundancy of the neuromechanical system also provides a rich landscape for motor variability (Ting and McKay, 2007) and perhaps motor style (Ting, 2007), creativity and improvisation (Chiel and Beer, 1997).

Recent research has demonstrated that the basic neural elements for producing movements are not individual muscles, but muscle synergies that coordinate multiple muscles in functionally meaningful ways. Muscle synergies may allow the body to be rapidly reconfigured in context-dependent ways (Flash and Hochner, 2005; Davidson et al., 2007; Ting, 2007; Ting and McKay, 2007). Like musical chords, each muscle synergy specifies how particular muscles (or notes) are concurrently activated. Just as one note belongs to several different chords, each muscle belongs to more than one muscle synergy (Fig. 2). When chords are played simultaneously, the underlying structure may no longer be evident in the multitude of notes.

In balance control, spatial patterns of muscle activity in response to multidirectional perturbations can be attributed to the activation of a few muscle synergies that produce consistent forces (Krishnamoorthy et al., 2003; Ting and Macpherson, 2005; Torres-Oviedo et al., 2006) or movement patterns (TorresOviedo and Ting, 2007). Moreover, trial-by-trial variability is constrained to the same spatial patterns, reflecting variations in the contributions of each muscle synergy in response to perturbations and not noise (Torres-Oviedo and Ting, 2007). Together with simple biomechanical models of balance control, these results suggest that the variations in the amplitude and temporal patterns of muscle activity are modulated by more abstract motor goals related to the control of the CoM and not of individual joints (Lockhart and Ting, 2007; Welch and Ting, 2008), habituation, and expectation (Torres-Oviedo and Ting, 2007).

Muscle synergies may provide rapid flexibility for motor adaptation, but may also constrain movement patterns. In both cats and humans, individual differences in muscle synergy patterns are consistently observed across days, yet the biomechanical functions they perform are similar (Torres-Oviedo et al., 2006; Torres-Oviedo and Ting, 2007). The same set of muscle has been shown to be used when the biomechanical context of the task is changed in cats (Torres-Oviedo et al., 2006). This suggests that each individual consistently prefers one solution from among many possible. In cats, the orientation of the resulting forces reflect both muscle synergy constraints (McKay and Ting, 2008) in addition to biomechanical properties of the limb (McKay et al., 2007). Computer simulations of human movement are able to capture individual movement styles by differentially constraining patterns of joint torques across the body (Liu et al., 2005).

Similar to concepts in sparse coding of sensory inputs (Olshausen and Field, 2004), across all motor tasks there may be far more muscle synergies than muscles (Fig. 2). However, within a given motor task, muscle synergies may reduce the number of variables modulated by hierarchical neural structures (Fiete et al., 2007; Hamed et al., 2007; Berniker et al., 2009), as well as provide a mapping between task-level variables and execution-level neural commands (Flash and Hochner, 2005; Ting and McKay, 2007), allowing rapid adaptation to novel situations. Similarly, in music, the number of available chords vastly outnumbers the number of notes on a scale, yet any particular composition is limited to a small set of chords that constrain the character of the composition independent of the temporal structure, and reflect the preferences of the composer.

Finally, the individual variations in muscle synergy patterns for balance may reflect the variable reliance on intrinsic musculoskeletal versus neural feedback control of balance as well as other factors. In a simple robotic model, modulation balance control dynamics can be equivalently achieved by varying biomechanical properties of the postural configuration or neural feedback control with realistic physiological delays (Scrivens et al., 2008). Moreover, altering tonic muscle stiffness can affect the required precision of the feedback response, and also whether the robot takes a step for balance recovery (Ting et al., 2009). Since different tonic patterns of muscle activity for standing can produce wide variations in the stability of the limb, individual variations in muscle synergies may be related to differences in the relative contributions of neural and mechanical stability in balance control.

More generally, variations in each individual's posture and balance control may reflect individual differences in how we manage our interactions with our physical, social, and psychological environments. While muscle synergies provide a quantitative and functional measure of the lowest level elements of motor coordination, much remains to be learned. A major challenge is to identify the neural correlates of these elements [see, for example, the studies by Schepens and Drew (2004), Schepens et al. (2008), Stapley and Drew (2009)]. What are the neuromechanical structures governing the hierarchal selection and modulation of muscle synergies during movement? How do these mechanisms develop, change, and degenerate over the life span? These questions will serve as the basis for exciting research over the next few years.

\section{Neuromechanical models of locomotion}

Reliable locomotion is crucial for survival, and this evolutionary pressure has resulted in mechanisms that are not only efficient but also highly redundant. For example, multiple muscles often produce torques around the same joint and multiple sense organs convey similar information. Redundancy is sometimes so strong that the nervous system and the body mechanics are both capable of generating locomotor movements on their own. Fictive locomotion (Brown, 1911) clearly demonstrates that the nervous system has an intrinsic capability of producing locomotor-like patterns even when deprived of its body. At the other extreme, passive walking devices (McGeer, 1990; Collins et al., 2005) are able to produce natural looking movements without any control system.

Fictive locomotion is an effective preparation for studying the neural basis of rhythmic behaviors (Grillner, 2006). Still, it must be remembered that locomotion without feedback is a very artificial situation and that important parts of the picture may be missed when the nervous system is studied in isolation. It is easy to get the impression that the neural networks are the sole origin of the motor patterns while the body only serves as a mediator, making the movements as dictated by the nervous system. Given the body's intrinsic abilities to produce suitable movements, it may be more appropriate to view the musculo-mechanical system and the neural system as a collaborating pair (Chiel and Beer, 1997). Understanding locomotion beyond the basic generation of rhythmic patterns, for example how stability is maintained and 
A

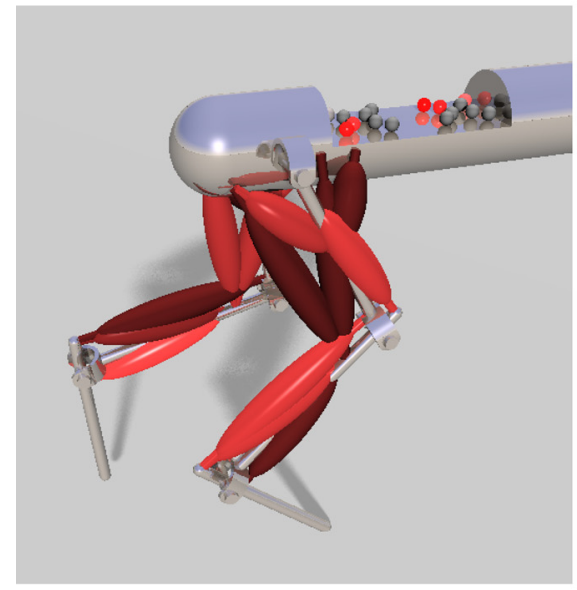

B

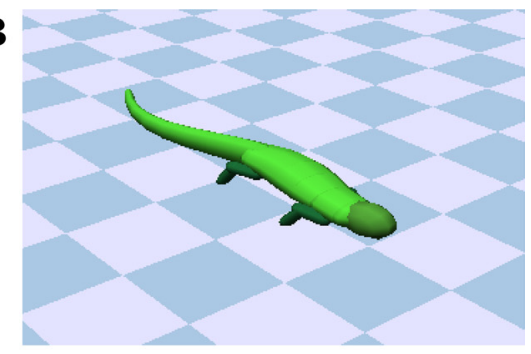

C

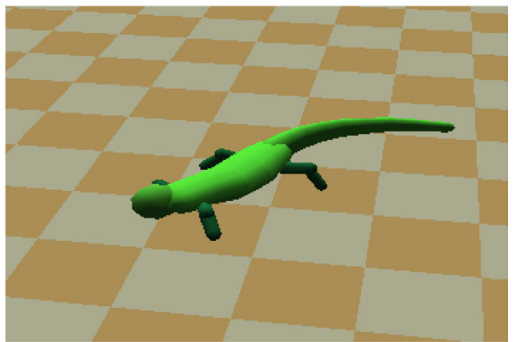

Figure 3. Simulations of walking and swimming in cat and salamander. $\boldsymbol{A}$, Snapshot from a simulation of the hindlegs of a walking cat model, where a neural network derived from the circuitry of the lamprey is used to activate the leg muscles. The color of the muscles indicates level of activation (red is high). $\boldsymbol{B}$, Screenshot from a neuro-mechanical simulation of a swimming salamander. Traveling neural waves produce body undulations which drive the body forward through the water. $\boldsymbol{C}$, The same salamander model driven with a motor pattern producing walking.

how we move under pathological conditions, requires a careful study of this interplay.

Traditional experimental techniques, in particular lesion and other blocking studies, are not effective in analyzing a system with redundancies since alternative mechanisms tend to take over, giving the impression that no part is crucial. Modeling via computer simulation and even using physical robots is one way to directly study the interplay between mechanisms. Skeletal mechanics and muscle properties are in principle straightforward to model mathematically, and when these models are coupled to models of the neuronal control system, aspects of what happens throughout the sensorymotor loop can be analyzed.

By augmenting a model of the lamprey spinal central pattern generator (CPG) with a muscle-and-body model placed in virtual water, a whole repertoire of behaviors such as turns, rolls, and backward and narrow swimming emerged without the need to add any extra neural circuitry (Ekeberg, 1993; Ekeberg et al., 1995). Regulation of muscle stiffness was found to be important, so that swimming occurs close to the mechanical resonance frequency, an observation that could not be made in the isolated nervous system.

Swimming in the lamprey constitutes one extreme in which sensory feedback is of relatively minor importance. Simulations of walking in stick insects can be seen as the opposite extreme, where sensory feedback plays a major role, not only for determining foot placement in a highly unstable and irregular environment, but also for coordinating legs and even individual joints that otherwise operate autonomously (Büschges et al., 1995; Borgmann et al., 2007). The observation that sensory feedback and leg coordination reflexes can generate gaits without major input from a central neural controller is not unique to insects. In the cat, mechanical coupling can also contribute to maintaining an alternating gait, provided that the CPGs adapt to the appropriate sensory cues (Fig. 3A). When a musculo-mechanical model of two cat hindlegs was driven by two state-based controllers, an alternating gait was actively maintained even without any crossed neural coupling, due to the stabilizing effect of leg load

sensors influencing the timing for stance termination (Ekeberg and Pearson, 2005).

A unique feature of simulations is that the same experiment can be repeated under exactly the same conditions. Making minute changes between runs enables the study of system-level effects of individual signals or mechanisms. This technique has been used to analyze how the musculomechanical system of the cat hind legs reacts to changes in neural output during ongoing walking (Harischandra and Ekeberg, 2008). The study reveals that during most parts of the step cycle, the system is perfectly damped to respond rapidly without the risk of excessive overshoots.

The salamander swims like a lamprey, but is also capable of walking. This animal has been modeled not only in simulations but also by building physical robots driven by CPG models based on the lamprey organization (Ijspeert et al., 2007). The transition between swimming and walking has attracted special interest, as it seems to rely on nontrivial interaction between axial (swimming) CPGs and specific leg CPGs. An ongoing collaborative project, LAMPETRA, aims to build robotic lampreys and salamanders with many natural properties (Fig. $3 B, C$ ). These robots, and simulations of them, are used as platforms for investigating various models of the neural control system. Eventually, motor output from real networks will be used to drive the robots in hybrid preparations, and may also receive sensory feedback to close the control loop. This will make it possible to preserve the advantages of fictive locomotion preparations while relaxing its major shortcoming: the lack of a body to interact with.

\section{Biomechanical constraints on sensing behaviors}

Locomotor movements, maintaining postural stability, and feeding behavior are all examples of movements that have an action or task as their end goal, and can be achieved with different spatiotemporal patterns of muscle activation. Biomechanical constraints are equally important during sensing and exploratory movements. During exploratory behavior, biomechanics constrain the movements that an animal can make, but the animal will exploit available degrees of freedom to improve the acquisition of desired sensory information.

The rat whisker (vibrissal) system is becoming an increasingly important model to study the role of biomechanics in sensing behaviors (Kleinfeld et al., 2006; Diamond et al., 2008). Rats are nocturnal animals with low acuity vision, and use 5-25 Hz movements of their whiskers to tactually extract object features, including size, shape, orientation, and texture. Approximately 30 whiskers are arranged in a regular array on each side of the rat's face. The base of each whisker is embedded within a densely innervated follicle where mechanoreceptors transduce deformations into electrical signals and provide input to primary sensory neurons in the trigeminal ganglion (Leiser and Moxon, 2006). Recent studies have combined mechanical experiments and simulation with behavioral studies to elucidate neuromechanical interactions at the level of the single whisker, as well as at the level of the entire head and whisker array. 
Considerable work has investigated the biomechanics of texture discrimination. Several recent studies on both artificial (wire) and real rat whiskers have demonstrated that whiskers undergo a series of "stick-slip-ring" events as they brush over a textured surface (Hipp et al., 2006; Lottem and Azouz, 2008; Ritt et al., 2008; Wolfe et al., 2008). The precise timing and mechanical details of stick-slip events depends on a complex interaction between texture properties, intrinsic whisker dynamics, and active control. The pattern of stick-slip timing is strongly texture dependent while the dependence of that pattern on intrinsic whisker dynamics is an open topic of investigation. The amplitude and frequency of ringing after a stick-slip event were found to be dependent on whisker identity but independent of texture roughness (Wolfe et al., 2008). The level of temporal precision that is behaviorally relevant to texture discrimination is a further open topic of investigation.

Other mechanical models have shown that the rat could determine the distance from the whisker basepoint to an object if neurons were to monitor the rate of change of moment (torque) at the whisker base. This mechanism for distance determination is consistent with those suggested by behavioral (Krupa et al., 2001) and neurophysiological (Szwed et al., 2006) studies. Based on the principle that rate of moment change is proportional to object distance, an array of mechanical whiskers was constructed that could extract precise information about three-dimensional object shape while the whiskers were either "tapping" or "sweeping" along an object (Solomon and Hartmann, 2006, 2008, 2009).

More recent work has investigated how the intrinsic curvature of a single whisker affects the forces that will be generated during object collision, specifically the axial ("longitudinal") force known to excite trigeminal ganglion neurons (Stüttgen et al., 2008). Forces at the whisker base were monitored during object collision in both the perpendicular and axial directions, schematized as $F_{\mathrm{x}}$ and $F_{\mathrm{y}}$ in Figure $4 A$. The magnitude of the axial force generated during collisions was found to be higher if the whisker collides with its convex side facing the object for small angles of impingement (Quist and Hartmann, 2008). Small angles of deflection are common in normal exploratory behavior, as described by the minimal impingement hypothesis of Michinson et al. (2007). In addition, during convex collisions, the rate of change of axial force could also provide the animal with a measure of radial object distance (Fig. $4 B$ ). This in turn leads to the behavioral prediction that the rat might sometimes alter its exploratory strategies to ensure convex collisions.

The work on postural stability above suggested that muscle synergies specify synchronous spatial patterns of muscle activation, but that temporal patterns are specified by a small set of task-level variables. There is evidence for a similar spatiotemporal decoupling in the rat vibrissal system. Recent high-speed video analysis revealed dramatic bilateral asymmetries and asynchronies in free-air whisking behavior (Towal and Hartmann, 2006). The spatial asymmetry was strongly correlated with rotational head velocity, ensuring a "look-ahead" distance of almost exactly one whisk. In contrast, bilateral asynchrony was large (up to $108 \mathrm{~ms}$ ), but only weakly dependent on head

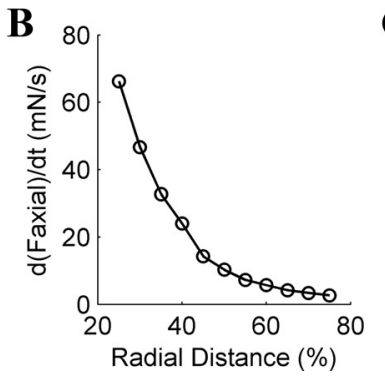

C

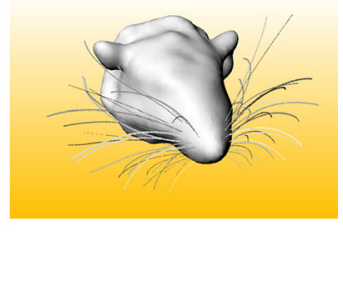
velocity. These results suggest that-unlike spatial asymme-
tries-temporal asynchrony is not constrained by head motion, and may be under more cognitive control, used for example to determine when a particular portion of an object will be sampled.

An ongoing project aims to integrate accurate whisker mechanics into a full three-dimensional simulation of the rat head and vibrissal array, illustrated in Figure 4C. Ultimately, the simulation will be used to predict the contact patterns-and resulting forces and moments-at each whisker base for a given exploratory sequence as the rat interacts with various objects in the environment.

\section{Summary and conclusions}

A brief review of the role of biomechanical and neural interactions in both motor and sensory behavior has revealed several common themes. It is clear that when the nervous system is interacting with the complex biomechanics of the body, and both are interacting with a complex and changing environment, features of behavior emerge that may not be clear when the components are studied in isolation. Understanding the relative roles of biomechanics and neural control, and the relative importance of feedforward and feedback control, may only be possible within this context. Moreover, unexpected simplifications of the control problem may result, especially if solutions emerge naturally from the mechanics of the periphery.

Several fruitful directions for further investigation are suggested by these studies. What dynamical structures may underlie sequential behavior that can be influenced by changing sensory inputs? Recent studies on dynamical structures that move systems from one equilibrium point to another along "channels" that can direct the trajectory of the system may be relevant to answering this question (Rabinovich et al., 2006). Bernstein's classic studies pose another question: he noted that motor "invariants" existed that were independent of the parts of the body that implemented them (Bernstein, 1967). For example, with practice, a person can generate a similar signature using a very fine pen under a magnifying glass or using a large paintbrush on canvas, which engages very different body parts to obtain the same final result. What could serve as the basis for "motor invariants"? Finally, the flexibility and robustness of motor and sensory systems may not only serve as the foundation on which higher cognitive functions are built, but may provide insights into the very nature of cognitive functions including the creation of an individual "style," the ability to modify behavior by learning, and the ability to combine novel sequences into entirely new behaviors. 


\section{References}

Berniker M, Jarc A, Bizzi E, Tresch MC (2009) Simplified and effective motor control based on muscle synergies to exploit musculoskeletal dynamics. Proc Natl Acad Sci U S A 106:7601-7606.

Bernstein N (1967) The coordination and regulation of movements. Oxford: Pergamon.

Bizzi E, Hogan N, Mussa Ivaldi FA, Giszter S (1992) Does the nervous system use equilibrium point control to guide single and multiple joint movements? Behav Br Sci 15:603-613.

Borgmann A, Scharstein H, Büschges A (2007) Intersegmental coordination: The role of a single walking leg for the activation of the stick insect walking system. J Neurophysiol 98:1685-1696.

Brown T (1911) The intrinsic factors in the act of progression in the mammal. Proc Biol Sci 84:308-319.

Bunderson NE, Burkholder TJ, Ting LH (2008) Reduction of neuromuscular redundancy for postural force generation using an intrinsic stability criterion. J Biomech 41:1537-1544.

Büschges A, Schmitz J, Bässler U (1995) Rhythmic patterns in the thoracic nerve cord of the stick insect induced by pilocarpine. J exp Biol 198:435-456.

Carpenter MG, Allum JH, Honegger F (1999) Directional sensitivity of stretch reflexes and balance corrections for normal subjects in the roll and pitch planes. Exp Brain Res 129:93-113.

Chiel HJ, Beer RD (1997) The brain has a body: Adaptive behavior emerges from interactions of nervous system, body and environment. Trends Neurosci 20:553-557.

Collins S, Ruina A, Tedrake R, Wisse M (2005) Efficient bipedal robots based on passive-dynamic walkers. Science 307:1082-1085.

Davidson AG, Chan V, O’Dell R, Schieber MH (2007) Rapid changes in throughput from single motor cortex neurons to muscle activity. Science 318:1934-1937.

Diamond ME, von Heimendahl M, Knutsen PM, Kleinfeld D, Ahissar E (2008) 'Where' and 'what' in the whisker sensorimotor system. Nat Rev Neurosci 9:601-612.

Ekeberg Ö (1993) A combined neuronal and mechanical model of fish swimming. Biol Cyber 69:363-374.

Ekeberg Ö, Pearson K (2005) Computer simulation of stepping in the hind legs of the cat: an examination of mechanisms regulating the stance-toswing transition. J Neurophysiol 94:4256-4268.

Ekeberg Ö, Grillner S, Lansner A (1995) The neural control of fish swimming studied through numerical simulations. Adapt Behav 3:363-384.

Elliott C, Susswein AJ (2002) Comparative neuroethology of feeding control in molluscs. J exp Biol 205:877-896.

Feldman AG, Latash ML (2005) Testing hypotheses and the advancement of science: recent attempts to falsify the equilibrium point hypothesis. Exp Brain Res 161:91-103.

Feldman AG, Ostry DJ, Levin MF, Gribble PL, Mitnitski AB (1998) Recent tests of the equilibrium-point hypothesis ( $\lambda$ model). Motor Control 2:189-205.

Fiete IR, Fee MS, Seung HS (2007) Model of birdsong learning based on gradient estimation by dynamic perturbation of neural conductances. J Neurophysiol 98:2038-2057.

Flash T, Hochner B (2005) Motor primitives in vertebrates and invertebrates. Curr Opin Neurobiol 15:660-666.

Grillner S (2006) Biological pattern generation: the cellular and computational logic of networks in motion. Neuron 52:751-766.

Hamed SB, Schieber MH, Pouget A (2007) Decoding M1 neurons during multiple finger movements. J Neurophysiol 98:327-333.

Harischandra N, Ekeberg Ö (2008) System identification of muscle-joint interactions of the cat hind limb during locomotion. Biol Cyber 99:125-138.

Hipp J, Arabzadeh E, Zorzin E, Conradt J, Kayser C, Diamond ME, König P (2006) Texture signals in whisker vibrations. J Neurophysiol 95:1792-1799.

Horak FB, Macpherson JM (1996) Postural orientation and equilibrium. In: Handbook of Physiology, Sec 12, pp 255-292. New York: American Physiological Society.

Ijspeert AJ, Crespi A, Ryczko D, Cabelguen JM (2007) From swimming to walking with a salamander robot driven by a spinal cord model. Science 315:1416-1420.

Jing J, Weiss KR (2001) Neural mechanisms of motor program switching in Aplysia. J Neurosci 21:7349-7362.
Jing J, Weiss KR (2002) Interneuronal basis of the generation of related but distinct motor programs in Aplysia: Implications for current neuronal models of vertebrate intralimb coordination. J Neurosci 22:6228-6238.

Katzoff A, Ben-Gedalya T, Hurwitz I, Miller N, Susswein YZ, Susswein AJ (2006) Nitric oxide signals that Aplysia have attempted to eat, a necessary component of memory formation after learning that food is inedible. J Neurophysiol 96:1247-1257.

Kleinfeld D, Ahissar E, Diamond ME (2006) Active sensation: Insights from the rodent vibrissa sensorimotor system. Curr Opin Neurobiol 16:435-444.

Krishnamoorthy V, Latash ML, Scholz JP, Zatsiorsky VM (2003) Muscle synergies during shifts of the center of pressure by standing persons. Exp Brain Res 152:281-292.

Krupa DJ, Matell MS, Brisben AJ, Oliveira LM, Nicolelis MA (2001) Behavioral properties of the trigeminal somatosensory system in rats performing whisker-dependent tactile discriminations. J Neurosci 21:5752-5763.

Kupfermann I, Carew TJ (1974) Behavioral patterns of Aplysia californica in its natural environment. Behav Biol 12:317-337.

Kupfermann I (1970) Stimulation of egg laying by extracts of neuroendocrine cells (bag cells) of abdominal ganglion of Aplysia. J Neurophysiol 33:877-881.

Kupfermann I (1974) Feeding behavior in Aplysia: a simple system for the study of motivation. Behav Biol 10:1-26.

Latash ML (2008) Evolution of motor control: from reflexes and motor programs to the equilibrium-point hypothesis. J Hum Kinetics 19:3-24.

Leiser SC, Moxon KA (2006) Relationship between physiological response type (RA and SA) and vibrissal receptive field of neurons within the rat trigeminal ganglion. J Neurophysiol 95:3129-3145.

Liu CK, Hertzmann A, Popovic Z (2005) Learning physics-based motion style with nonlinear inverse optimization. ACM Trans Graph 24:10711081.

Lockhart DB, Ting LH (2007) Optimal sensorimotor transformations for balance. Nat Neurosci 10:1329-1336.

Lottem E, Azouz R (2008) Dynamic translation of surface coarseness into whisker vibrations. J Neurophysiol 100:2852-2865.

Lu H, Chestek CA, Shaw KM, Chiel HJ (2008) Selective extracellular stimulation of individual neurons in ganglia. J Neural Eng 5:287-309.

McGeer T (1990) Passive dynamic walking. Int J Robotics Res 9:62-82.

McKay JL, Burkholder TJ, Ting LH (2007) Biomechanical capabilities influence postural control strategies in the cat hindlimb. J Biomech 40:2254-2260.

McKay JL, Ting LH (2008) Functional muscle synergies constrain force production during postural tasks. J Biomech 41:299-306.

Mitchinson B, Martin CJ, Grant RA, Prescott TJ (2007) Feedback control in active sensing: rat exploratory whisking is modulated by environmental contact. Proc Biol Sci 274:1035-1041.

Morton DW, Chiel HJ (1993a) In vivo buccal nerve activity that distinguishes ingestion from rejection can be used to predict behavioral transitions in Aplysia. J Comp Physiol A 172:17-32.

Morton DW, Chiel HJ (1993b) The timing of activity in motor-neurons that produce radula movements distinguishes ingestion from rejection in Aplysia. J Comp Physiol A 173:519-536.

Nagengast AJ, Braun DA, Wolpert DM (2009) Optimal control predicts human performance on objects with internal degrees of freedom. PLoS Comput Biol 5:e1000419.

Nashner LM (1976) Adapting reflexes controlling the human posture. Exp Brain Res 26:59-72.

Nashner LM (1977) Fixed patterns of rapid postural responses among leg muscles during stance. Exp Brain Res 30:13-24.

Neustadter DM, Drushel RF, Chiel HJ (2002a) Kinematics of the buccal mass during swallowing based on magnetic resonance imaging in intact, behaving Aplysia californica. J Exp Biol 205:939-958.

Neustadter DM, Drushel RF, Crago PE, Adams BW, Chiel HJ (2002b) A kinematic model of swallowing in Aplysia californica based on radula/ odontophore kinematics and in vivo magnetic resonance images. J Exp Biol 205:3177-3206.

Neustadter DM, Herman RL, Drushel RF, Chestek DW, Chiel HJ (2007) The kinematics of multifunctionality: comparisons of biting and swallowing in Aplysia californica. J exp Biol 210:238-260.

Novakovic VA, Sutton GP, Neustadter DM, Beer RD, Chiel HJ (2006) Mechanical reconfiguration mediates swallowing and rejection in Aplysia 
californica. J Comp Physiol A Neuroethol Sens Neural Behav Physiol 192:857-870.

Olshausen BA, Field DJ (2004) Sparse coding of sensory inputs. Curr Opin Neurobiol 14:481-487.

Quist B, Hartmann M (2008) Vibrissal mechanics: relations between inherent whisker curvature, axial force, and radial distance determination. $\mathrm{Pa}$ per presented at Barrels 2008. October, Washington, DC.

Rabinovich M, Varona P, Selverston A, Abarbanel H (2006) Dynamical principles in neuroscience. Rev Mod Phys 78:1213-1265.

Ritt JT, Andermann ML, Moore CI (2008) Embodied information processing: Vibrissa mechanics and texture features shape micromotions in actively sensing rats. Neuron 57:599-613.

Schepens B, Drew T (2004) Independent and convergent signals from the pontomedullary reticular formation contribute to the control of posture and movement during reaching in the cat. J Neurophysiol 92:2217-2238.

Schepens B, Stapley P, Drew T (2008) Neurons in the pontomedullary reticular formation signal posture and movement both as an integrated behavior and independently. J Neurophysiol 100:2235-2253.

Scrivens JE, Deweerth SP, Ting LH (2008) A robotic device for understanding neuromechanical interactions during standing balance control. Bioinspir Biomim 3:26002.

Solomon JH, Hartmann MJ (2006) Sensing features with robotic whiskers. Nature 443:525.

Solomon J, Hartmann M (2008) Artificial whiskers suitable for array implementation: Accounting for lateral slip and surface friction. IEEE Trans Robotics Automat 24:1157-1167.

Solomon J, Hartmann M (2009) Extracting object contours with the sweep of a robotic whisker using torque information. Int $J$ Robotics Res. Advance online publication. Retrieved September 13, 2009. doi:10.1177/0278364908104468.

Stapley PJ, Drew T (2009) The pontomedullary reticular formation contributes to the compensatory postural responses observed following removal of the support surface in the standing cat. J Neurophysiol 101:1334-1350.

Strogatz SH (1994) Nonlinear dynamics and chaos. Reading, MA: Perseus Books.

Stüttgen MC, Kullmann S, Schwarz C (2008) Responses of rat trigeminal ganglion neurons to longitudinal whisker stimulation. J Neurophysiol 100:1879-1884.

Sutton GP, Mangan EV, Neustadter DM, Beer RD, Crago PE, Chiel HJ (2004) Neural control exploits changing mechanical advantage and context dependence to generate different feeding responses in Aplysia. Biol Cybern 91:333-345.
Szwed M, Bagdasarian K, Blumenfeld B, Barak O, Derdikman D, Ahissar E (2006) Responses of trigeminal ganglion neurons to the radial distance of contact during active vibrissal touch. J Neurophysiol 95:791-802.

Ting LH (2007) Dimensional reduction in sensorimotor systems: a framework for understanding muscle coordination of posture. Prog Brain Res 165:299-321.

Ting LH, Macpherson JM (2004) Ratio of shear to load ground-reaction force may underlie the directional tuning of the automatic postural response to rotation and translation. J Neurophysiol 92:808-823.

Ting LH, Macpherson JM (2005) A limited set of muscle synergies for force control during a postural task. J Neurophysiol 93:609-613.

Ting LH, McKay JL (2007) Neuromechanics of muscle synergies for posture and movement. Curr Opin Neurobiol 17:622-628.

Ting LH, van Antwerp KW, Scrivens JE, McKay JL, Welch TD, Bingham JT, DeWeerth SP (2009) Neuromechanical tuning of nonlinear postural control dynamics. Chaos 19:026111.

Torres-Oviedo G, Ting LH (2007) Muscle synergies characterizing human postural responses. J Neurophysiol 98:2144-2156.

Torres-Oviedo G, Macpherson JM, Ting LH (2006) Muscle synergy organization is robust across a variety of postural perturbations. J Neurophysiol 96:1530-1546.

Towal RB, Hartmann MJ (2006) Right-left asymmetries in the whisking behavior of rats anticipate head movements. J Neurosci 26:8838-8846.

van Antwerp KW, Burkholder TJ, Ting LH (2007) Inter-joint coupling effects on muscle contributions to endpoint force and acceleration in a musculoskeletal model of the cat hindlimb. J Biomech 40:3570-3579.

Welch TD, Ting LH (2008) A feedback model reproduces muscle activity during human postural responses to support-surface translations. J Neurophysiol 99:1032-1038.

Wolfe J, Hill DN, Phlavan S, Drew PJ, Kleinfeld D, Feldman DE (2008) Texture coding in the rat whisker system: slip-stick versus differential resonance. PLoS Biol 6:e215.

Ye H, Morton DW, Chiel HJ (2006a) Neuromechanics of coordination during swallowing in Aplysia californica. J Neurosci 26:1470-1485.

Ye H, Morton DW, Chiel HJ (2006b) Neuromechanics of multifunctionality during rejection in Aplysia californica. J Neurosci 26:10743-10755.

Zajac FE, Gordon ME (1989) Determining muscle's force and action in multi-articular movement. Exerc Sport Sci Rev 17:187-230.

Zajac FE (1993) Muscle coordination of movement: a perspective. J Biomech 26 [Suppl 1]:109-124. 